\title{
2 I 28 Free-breathing steady-state free precession 3D coronary MRA: comparison of diaphragm and cardiac fat navigator techniques
}

\author{
Thanh D Nguyen*, Pascal Spincemaille, Martin R Prince and Yi Wang
}

Address: Weill Medical College of Cornell University, New York, NY, USA

* Corresponding author

from I th $^{\text {th }}$ Annual SCMR Scientific Sessions

Los Angeles, CA, USA. I-3 February 2008

Published: 22 October 2008

Journal of Cardiovascular Magnetic Resonance 2008, I0(Suppl I):A397 doi:I0.I I86/I532-429X-I0-SI-A397

This abstract is available from: http://jcmr-online.com/content//0/SI/A397

(c) 2008 Nguyen et al; licensee BioMed Central Ltd.

\section{Introduction}

Physiologic motion presents a major challenge in highresolution coronary MRA (CMRA). Current free-breathing 3D CMRA employs diaphragm navigator gating (DNAV) for respiratory motion suppression, where the respirationinduced cardiac motion is estimated indirectly from the diaphragmatic motion. When the correlation between diaphragmatic and cardiac motions is poor due to hysteresis, the effectiveness of DNAV gating is suboptimal. To overcome this limitation, the cardiac fat navigator (FNAV) was developed to provide a direct measurement of bulk cardiac motion by selectively exciting the epicardial fat. A preliminary study suggested that FNAV provides equal or more effective motion suppression than DNAV for SPGR 3D CMRA, but the statistical significance of the image quality improvement was not conclusive. Furthermore, balanced SSFP imaging has recently replaced SPGR imaging as the state-of-the-art sequence for 3D CMRA.

\section{Purpose}

The objective of this study was to compare the performance of DNAV and FNAV in free-breathing SSFP 3D CMRA and to determine the statistical significance of the differences in image quality, SNR, CNR, and navigator efficiency between the two techniques.

\section{Methods}

Figure 1 shows the schematics of the implemented ECGtriggered free-breathing CMRA. AP and RL spatial saturation pulses were used to suppress the chest wall signal (including fat) for the subsequent FNAV. FNAV consisted of a 16-ms spectrally selective RF to excite the epicardial fat followed by cranial-caudal k-space sampling to monitor cardiac motion. DNAV consisted of a 2D selective pencil-beam RF to monitor the cranial-caudal motion of the dome of the right hemi-diaphragm.

Imaging was performed in eleven volunteers (10 men, mean age of $32 \pm 10$ years) using a $1.5 \mathrm{~T}$ GE commercial scanner. The typical imaging parameters were: TR/TE/FA/ $\mathrm{rBW}=4.0 \mathrm{~ms} / 1.5 \mathrm{~ms} / 60^{\circ} / \pm 62.5 \mathrm{kHz}$, resolution $=1.0 \times$

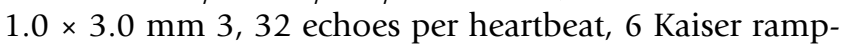
up SSFP preparation. A custom gating program was implemented to collect navigator data, extract motion information, and control data acquisition in real time. The phase ordering with automatic window selection (PAWS) gating algorithm, which optimizes gating window selection even in the case of respiratory drift and minimizes residual

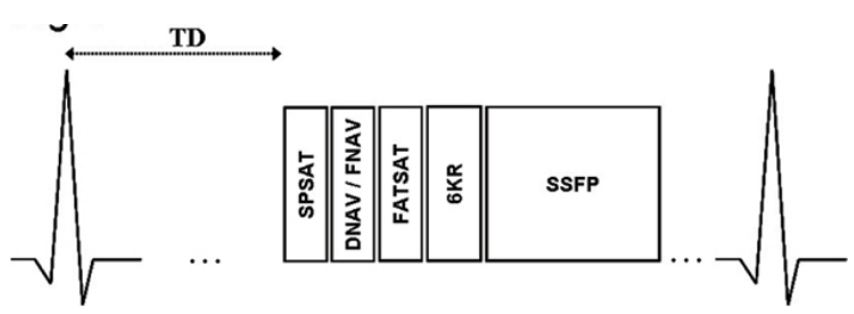

Figure I 


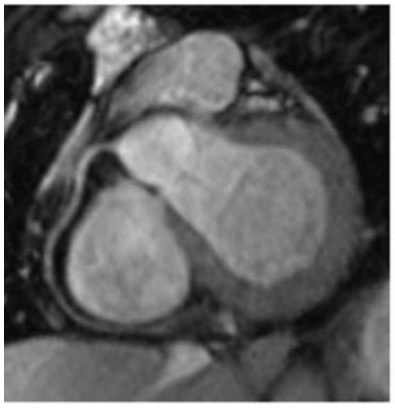

DNAV

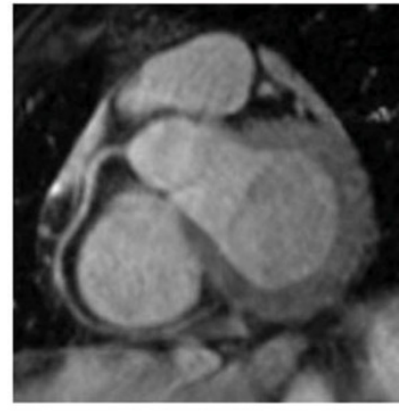

FNAV
Figure 2

motion artifacts within the gating window, was used with a gating window of $5 \mathrm{~mm}$ for DNAV and $2.5 \mathrm{~mm}$ for FNAV (corresponding to a linear correlation factor of 0.5 as reported previously). The DNAV and FNAV coronary sequences were performed in random order. Image quality difference was assessed by three experienced readers using randomized image pairs (five-point scale: markedly better, moderately better, similar, moderately worse, and markedly worse). The scores were combined with differences resolved by majority. Blood SNR and blood-myocardium CNR were also calculated using ROI analysis.

\section{Results}

Compared to DNAV gating, FNAV gating provided markedly better image quality in four subjects, moderately better quality in two subjects, and similar quality in six subjects $(\mathrm{P}<0.05)$. Diagnostically interpretable CMRA was obtained in all eleven subjects with FNAV gating ( $0 \%$ failure rate) and only nine subjects with DNAV gating (18\% failure rate). Figure 2 illustrates a case where DNAV and FNAV gating techniques produced similar image qualities of the RCA with negligible motion artifacts. Figure 3 shows FATNAV more effectively suppressing motion

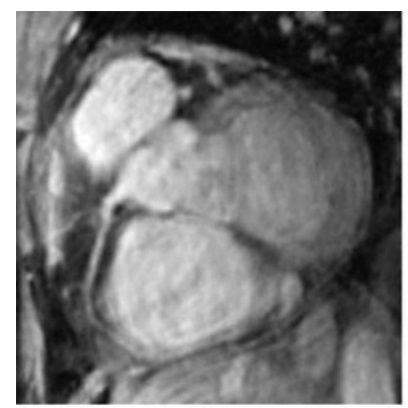

DNAV

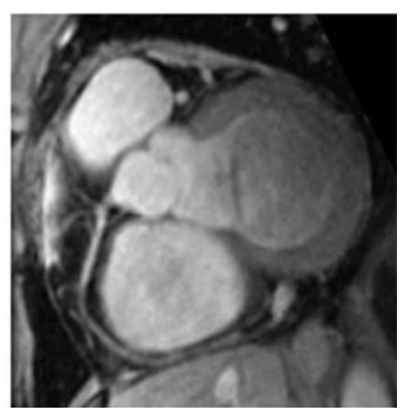

FNAV
Figure 3

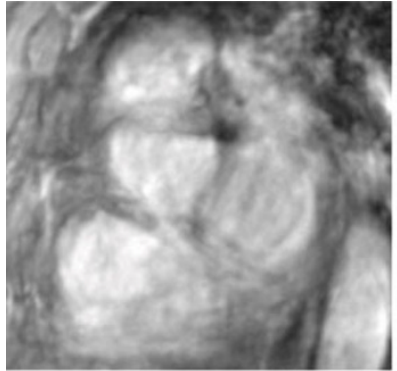

DNAV

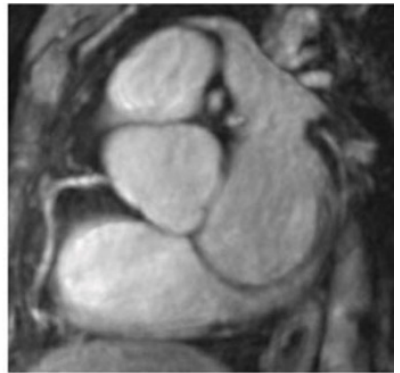

FNAV
Figure 4

than DNAV, leading to superior visualization of the RCA and better overall image quality. When DNAV gating failed, severe ghosting and blurring of the coronary arteries and the cardiac chambers were observed, but corresponding FNAV gating provided images with effective motion suppression (Figure 4). While minor differences in SNR $(P=0.8)$ and CNR $(P=0.19)$ were not statistically significant, FATNAV provided a $31 \%$ improvement in average navigator efficiency compared to DNAV $(\mathrm{P}=$ 0.02).

\section{Conclusion}

Cardiac fat navigator gating provides more effective motion suppression and better image quality than diaphragm navigator gating for free-breathing balanced SSFP 3D coronary MRA. 